\title{
Situational Analysis of Management of Childhood Diarrhea and Pneumonia in 13 District Hospitals in India
}

\author{
Harish Kumar, ${ }^{1}$ Ashfaq Ahmed Bhat, ${ }^{2}$ Varun Alwadhi,${ }^{3}$ Rajat Khanna, ${ }^{2}$ Sutapa B Neogi, ${ }^{4}$ AJay Khera ${ }^{5}$ And \\ SILA DEB ${ }^{5}$ \\ ${ }^{1}$ Vriddhi, IPE Global Ltd., New Delhi, India; ${ }^{2}$ Norway India Partnership Initiative, New Delhi, India; ${ }^{3}$ Kalawati Saran Children \\ Hospital, New Delhi, India; ${ }^{4}$ International Institute of Health Management Research, New Delhi, India; and ${ }^{5}$ Ministry of Health and \\ Family Welfare, Government of India, New Delhi, India. \\ Correspondence to: Dr Ashfaq Ahmed Bhat, Norway India Partnership Initiative, New Delhi, India.bhatashfaq@gmail.com \\ Received: May 08, 2019; Initial review: October 04, 2019; Accepted: September 28, 2020.
}

\begin{abstract}
Objective: To generate evidence on the current situation of hospital care (emergency, inpatient and outpatient), for managing children presenting with diarrhea and pneumonia at 13 district hospitals in India.

Design: Facility-based assessment of district hospitals.

Settings: 13 district hospitals in four states of Bihar, Madhya Pradesh, Odisha and Rajasthan.

Participants: Staff nurses and doctors.

Intervention: None.

Methods: An assessment was done across 13 district hospitals in four states by a group or trained assessors using an adapted quality assurance tool developed by Government of India where each aspect of care was scored (maximum score 5). Emergency services and triage, case management practices, laboratory support, and record maintenance for diarrhea and pneumonia were assessed.
\end{abstract}

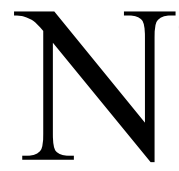
ewborn conditions, diarrhea and pneumonia contribute to nearly $79.4 \%$ of deaths in Indian children under five years of age [1]. The Integrated Global Action Plan for Prevention and Management of Pneumonia and Diarrhoea (GAPPD), 2013 includes treatment of sick children at health facilities as an integral component of the three pronged approach (protect, prevent, and treat) [2].

Delivery of good quality facility-based healthcare can reduce childhood deaths in low and middle-income countries where mortality is high. Both anecdotal and empirical evidence suggest that the quality of services can be improved only when it is reliably measured [3-5]. Various studies of the quality of services given to children in developing countries have highlighted deficiencies in practice of pediatric clinical guidelines, health worker training, triage and emergency treatment systems, staffing, monitoring, inadequate supportive care, essential equipment and medication [5-7]. A
Results: Separate diarrhea treatment unit was not earmarked in any of the DHs surveyed. Overall score obtained for adequate management of diarrhea and pneumonia was 2 and 2.2 which were poor. Pediatric beds were $6.8 \%$ of the total bed strength against the recommended $8-10 \%$. There was a 65 percent shortfall in the numbers of medical officers in position and 48 percent shortfall of nurses. There were issues with availability and utilization of drugs and equipment at appropriate places with cumulative score of 2.8. Triage for sick children was absent in all the facilities.

Conclusion: The standards of pediatric care for management of diarrhea and pneumonia were far from satisfactory. This calls for improvement of pediatric care units and implementation of operational guidelines for improving management of diarrhea and pneumonia.

Keywords: Assessment, Childhood illness, Operational guidelines, Quality assurance.

Published online: January 02, 2021; PII: S097475591600272

qualitative study in 13 district hospitals and eight teaching hospitals in seven less-developed countries highlighted that 14 of 21 hospitals lacked an adequate system for triage. Initial patient assessment was often inadequate and treatment delayed [8].

Editorial commentary: Pages 313-14

In India, as an adaptation of GAPPD, the Government of India (GoI) along with WHO and UNICEF developed an Integrated Action Plan for Pneumonia and Diarrhea (IAPPD) [9] in September, 2014. The district hospital (DH) is positioned in this continuum as second referral health facility, which provides specialist care under National Health Mission (NHM). The DH is envisaged as the hub of effective curative care for children, including those in age group 0-5 years, for common morbidities like diarrhea and pneumonia.

There is a dearth of evidence on the current situation 
of hospital care (emergency, inpatient and outpatient) for managing children presenting with diarrhea and pneumonia at DHs in India. Norway India Partnership Initiative (NIPI), launched in 2006, was a collaborative effort between Governments of Norway and India to support the NHM by providing strategic and catalytic support, and by testing scalable health system innovations. As part of this initiative, a comprehensive assessment was conducted across five states that highlighted gaps in the basic requirements for delivering effective pediatric care. The current study aims to assess the secondary health facilities (district hospitals) for provision of services with focus on management of diarrhea and pneumonia as part of Facility-based Integrated Management of Neonatal and Childhood Illness (F-IMNCI).

\section{METHODS}

This cross sectional study was undertaken as part of NIPI newborn project in May, 2014 across DHs of 13 implementation districts in four states under the project (2013-2017) that contribute to maximum childhood mortality: Bihar, Odisha, Madhya Pradesh and Rajasthan. The districts had a total population of more than two crores with an estimated under five population of 27 lakhs, and 4.82 lakh estimated live births each year.

A team of trained doctors (assessors) oriented on the protocol visited the hospital after prior information. Cases were observed to assess clinical management of children admitted to hospitals with diarrhea and pneumonia during the visit. This was also complemented by a review of case records. Observations were largely focused on understanding delivery of standard care for diarrhea and pneumonia, as information was inadequate in patient records. Clarifications were sought from doctors and nurses wherever required. Mothers/caregivers were also interviewed.

The assessment tool was adapted from the quality assurance tool developed by the Ministry of Health and Family Welfare (MoHFW), GoI [10]. It comprised of 12 sections including general hospital information, hospital support systems, organization of the emergency care and children's ward, case management of childhood diseases, supportive care, monitoring and hospital administration. Each section included a range of questions including those pertaining to case management. Scores were given based on whether the $\mathrm{DH}$ met the national standards for management of diarrhea and pneumonia, as detailed under F-IMNCI. Provisions were made to record personal observations if any. The adequacy of bed strength were assessed by Indian Public Health Standards according to which 300 beds should be available for 10 lakh population. Similarly, adequacy of number of medical officers, pediatricians and nurses were also assessed [11].

Scoring from 1 to 5 for each point was done comparing with standard of care, where 1 was for services not provided, totally inadequate care or potentially lifethreatening practices requiring urgent improvement, 2 for considerable need for improvement, 3 for some need for improvement, 4 for little need for improvement, and 5 for good practice complying with standards of care. Summary scores of all DHs were calculated for a cumulative score.

Data were collected on paper forms. Completed assessment tools from all the assessors were received at the state and national offices of UNDP-NIPI newborn project and scrutinized for data discrepancy by the monitoring and evaluation unit. Data were entered and compiled at state and national offices in a Microsoft Excel format. SPSS was used for analysis of data.

Approval for health facility assessment and data collection was obtained from MoHFW, GoI and the state governments. Since quality assurance is part of the government policy, ethical approval for this particular activity was not sought from any ethics committee. However, the identity of staff and care givers interviewed during the assessment was kept confidential and personal identifiers were not recorded. Appropriate feedback was given to the district and state authorities after the assessment.

\section{RESULTS}

All 13 districts had a functioning DH with number of beds ranging from 100 to 487 . A total of 3171 functional hospital beds were available with 216 (6.8\%) paediatric beds. Additional 76 beds in nutritional rehabilitation centres and 155 beds in sick newborn care units were also available. The total bed strength at DHs fell short by $50 \%$. There was a $65 \%$ shortfall in the numbers of medical officers in position and $48 \%$ shortfall of nurses. A $36 \%$ shortfall of pediatricians was recorded, which would be higher after accounting for pediatricians who were allocated to other units simultaneously. Only six pediatricians were trained in F-IMNCI, two medical officers were trained in advanced life support, and none of the other staff working in emergency department were trained in Emergency Triage and Treatment (ETAT). However, all children were attended by atleast MBBS doctors.

All the four DHs in Madhya Pradesh had continuous electricity supply and power backup was available only in emergency and not the pediatric wards or OPDs in Rajasthan, Odisha and Bihar. Running water was 


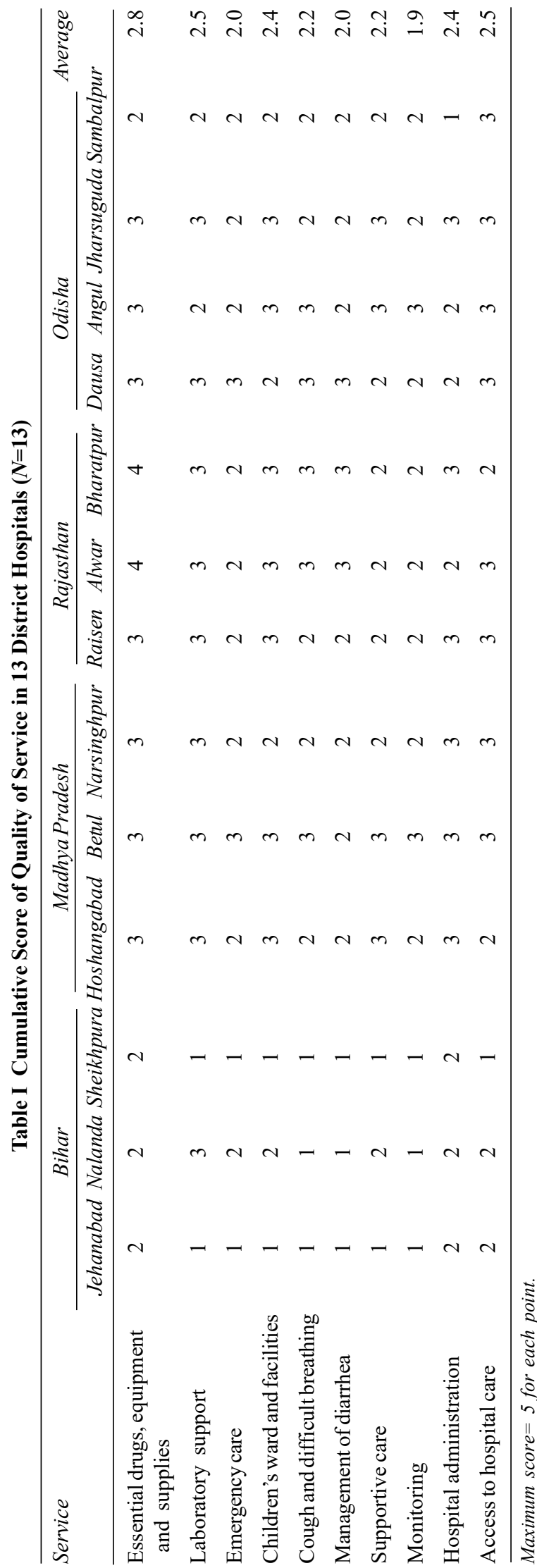

available round-the-clock in nine districts in the emergency, while only seven had running water available in pediatric ward. Biomedical waste management was inadequate across DHs, more so in Rajasthan and Bihar, where all the DHs lacked proper segregation of waste.

Laboratory support was available across all DHs, except one. The existing laboratory services needed improvement in terms of round-the-clock availability and the range of diagnostic tests performed. All DHs were found to be providing inadequate supportive care as shown in Table I. There were no major financial barriers with regard to the availability of essential drugs and transportation. However, caregivers were not aware of the provision of free transportation facilities for sick newborns and infants from home to health facility (under JSSK scheme). Referral transport was most often used for referrals between health facilities than for transport of sick children from home to health facility.

Separate pediatric wards were present in 12 out of 13 DHs surveyed. None of the DHs had a system for triage for sick children, though they had a separate area/department for managing emergencies. Staffing of emergency department in all DHs was limited to one medical officer and one nurse in each of the three shifts.

The performance of DHs in management of pneumonia and diarrhea are shown in Table II. Irrational use of antibiotics, corticosteroids, sedatives and antihistamines was evident; use of third generation cephalosporin was common instead of penicillin derivatives plus aminoglycoside combination, as recommended. Nebulizers and salbutamol respiratory solution were available in pediatric ward in seven and five DHs, respectively; however, their use was not observed during the visit. Regular monitoring of admitted patients and records of examined patients were not maintained for cross verification. In nine DHs, most of the admitted children were administered IV fluids, even when not indicated, and used as a calorie source beyond initial phase of stabilization.

None of the DHs had a separate area or Diarrhea Treatment Unit (DTU) or provision for performing tests for electrolytes (sodium and potassium levels) or stool culture. Dehydration was not correctly assessed or recorded. Use of ORS in children admitted to DH was not observed and intravenous fluids were being administered to all children. Interviews with mothers revealed that feeding history was not assessed and advised. Ageappropriate food was not served to sick inpatient children in five DHs. Records of inpatients for clinical progress, monitoring, feeding or medications administered were not maintained. Standard patient record formats were not 
Table II Performance of District Hospitals in the Management of Pneumonia and Diarrhea $(N=10)$ *

\begin{tabular}{lr}
\hline $\begin{array}{l}\text { Standards and criteria } \\
\text { for management }\end{array}$ & $\begin{array}{l}\text { No. of facilities } \\
\text { to be improved }\end{array}$ \\
\hline
\end{tabular}

For cough or difficult breathing

Severity of pneumonia is correctly assessed and diagnosed.

Appropriate antibiotics are administered for pneumonia and other respiratory diagnoses

Oxygen is correctly administered when necessary

Correct use of chest $X$-ray

Appropriate diagnosis and management of TB

Inhaled bronchodilators are given appropriately when indicated

Patient monitoring appropriately performed and charted

Supportive care provided appropriate for condition

For diarrhea

Dehydration is correctly assessed

Rehydration plan is appropriate to severity of

dehydration, and appropriately monitored

Appropriate antibiotics only given when necessary

Antidiarrheal drugs not given

Zinc used for managing diarrhea

Appropriate (continued) feeding given during diarrhea Corticosteroids are only given for a clear indication

*Data not included for Jehanabad, Nalanda, and Sheikhpura as there were no inpatients.

used or remained uncharted. Re-assessment of a child admitted during routine hours by a doctor was unlikely. Nutritional assessment was not undertaken in outpatients and inpatients routinely, therefore there were few referrals or admissions to nutritional rehabilitation centers (co- located at DH at many sites).

\section{DISCUSSION}

An assessment of $13 \mathrm{DHs}$ in four states showed that the basic infrastructure for managing cases of diarrhea and pneumonia and supportive services were present with shortage of human resources. Cumulative score for each service component across every facility was less than satisfactory with deficits in clinical case management, record maintenance and triage.

There were certain limitations on the assessment of DHs for management of pediatric diarrhoea and pneumonia. We did not consider all the elements of quality of health care viz. effectiveness, safety, people centricity, timeliness, equity, integrated services and efficiency, since this was beyond the scope of the assessment and the tool used. Direct observation of admitted cases was carried out but ongoing evaluation as outlined in the F-IMNCI protocol could not be done due to logistic issues. The skills of doctors and nurses were not assessed. The study was restricted to $13 \mathrm{DHs}$ in high burden states; hence, generalizability of findings across all settings becomes difficult.

The assessment findings are comparable to the evidence generated from other low and middle income countries (LMICs) [5,7,8,12]. Shortage of beds was reported consistently from all sites in the present study, which is likely to influence the patient outcomes [13]. Many DHs run by under-resourced non-government organizations like in remote parts of Papua New Guinea [12] and sub-district hospitals in Bangladesh [5], have general wards to house both adults and children together, unlike most DHs in the present assessment. Assessment of emergency services in primary and secondary level facilities indicate the strength and efficiency of the health system [14]. A functional triage seems to be a challenge in many hospitals in LMICs [4,5]. Similarly, none of the DHs had DTU that is a requirement as laid down by facilitybased pediatric operational guidelines [15]. The absence of these facilities is likely to compromise with the quality of services and can be related to poor outcomes such as mortality.

Case management practices from LMICs highlight certain challenges, similar to the findings that have emerged from our assessment. Inadequacies were seen in key tasks such as prescription of antibiotics and feeds even when resources were made available and guidelines provided $[5,16]$. This could be because of gaps in skill building of staff as also seen in experience from Papua New Guinea [12] and Malawi [16]. Quality of services for pneumonia was better in urban hospitals in Rwanda compared to rural hospitals [7]. In a similar nation-wide study conducted on case management of pneumonia in DHs in Malawi, case management guidelines were poorly implemented with frequent interruptions in the supply of antibiotics, oxygen, and lack of a reporting system [8]. Availability of essential drugs was seen to be a challenge in Bangladesh [5] similar to our study.

Documentation of clinical management remains a challenge in the assessment of DHs. A retrospective record review from Kenya showed missed outcome details in $20 \%$ of the admissions [19]. Clinical documentation of signs and symptoms was also poor in eight DHs in Kenya [16]. Training of staff on ETAT and management of diarrhoea and pneumonia resulted in improvement of practices and implementation of guidelines but documentation remained poor [20]. Even with minimum investment, triage based on the risk scores can 


\section{WHAT THIS STUDY ADDS?}

- Standards and quality of services were found to be unsatisfactory in 13 district hospitals across four states in India with need to upscale manpower and training of healthcare providers.

inform decisions [21]. With no additional investment of material resources, face to face feedback of performance, supportive supervision and provision of a local facilitator resulted in improvements of pediatric care and the readiness for providing care at DHs [22]. In most countries, academic rigour is directed towards clinical care in tertiary university hospitals with inadequate attention to peripheral hospitals, which are understaffed, poorly equipped and maintained, and have limited staff training [23].

To conclude, the assessment showed that standards of care for management of diarrhea and pneumonia were far from being met. It calls for planning and systematic implementation of the following recommendations: establishment of pediatric triage in all districts hospitals to facilitate rational management depending on the severity of diarrhea and pneumonia, based on national standards and operational guidelines, development of skills of doctors and nurses to manage pediatric emer-gencies (ETAT), management of diarrhea and pneumonia as per national standards of care (F-IMNCI); improve-ment of record keeping for pediatric services, both inpatient and outpatient, and introduction of standard patient record formats.

Contributors: HK: conceptualized the study and provided overall guidance; AAB,VA: guided the data compilation and supervised different steps involved in the study; RK: compiled and analyzed data of the assessment and supported the drafting of manuscript; SBN: reviewed the literature and drafted the manuscript; HK, AAB, AK, SD: technical inputs in finalizing the manuscript. All the authors have approved the final version of the manuscript.

Funding: Norway India Partnership Initiative (NIPI). Competing interest: None stated.

\section{REFERENCES}

1. Liu L, Oza S, Hogan D, et al. Global, regional, and national causes of child mortality in $2000-13$, with projections to inform post-2015 priorities: An updated systematic analysis. Lancet. 2015;385:430-40.

2. Chan M, Lake A. Integrated action for the prevention and control of pneumonia and diarrhoea. Lancet. 2013;381: 1436-7.

3. Ntoburi S, Hutchings A, Sanderson C, Carpenter J, Weber M, English M. Development of paediatric quality of inpatient care indicators for low-income countries - A Delphi study. BMC Pediatr. 2010;10:90.

4. Hategeka C, Mwai L, Tuyisenge L. Implementing the Emergency Triage, Assessment and Treatment plus admission care $(\mathrm{ETAT}+)$ clinical practice guidelines to improve quality of hospital care in Rwandan district hospitals: healthcare workers' perspectives on relevance and challenges. BMC Hlth Services Res. 2017;17:256.

5. Hoque DME, Rahman M, Billah SM, et al. An assessment of the quality of care for children in eighteen randomly selected district and sub-district hospitals in Bangladesh. BMC Pediatr. 2012;12:197.

6. Campbell H, Duke T, Weber M, et al. Global initiatives for improving hospital care for children: state of the art and future prospects. Pediatrics. 2008;121:e984-e92.

7. Hategeka C, Shoveller J, Tuyisenge L, Kenyon C, Cechetto DF, Lynd LD. Pediatric emergency care capacity in a lowresource setting: An assessment of district hospitals in Rwanda. PLoS One. 2017;12:e0173233.

8. Nolan T, Angos P, Cunha AJ, et al. Quality of hospital care for seriously ill children in less-developed countries. Lancet. 2001;357:106-10.

9. Ministry of Health and Family Welfare. India Newborn Action Plan (INAP) New Delhi: Ministry of Health and Family Welfare; 2013 [cited 2019 Feberuary 12].

10. Ministry of Health and Family Welfare. Operational guidelines for Quality Assurance in public health facilities New Delhi. 2013 [cited 2019 July 15].

11. Directorate General of Health Services. Indian Public Health Standards (IPHS)- Guidelines for District Hospitals New Delhi 2012. [cited 2019 March 3].

12. English M, Esamai F, Wasunna A, et al. Assessment of inpatient paediatric care in first referral level hospitals in 13 districts in Kenya. Lancet. 2004;363:1948-53.

13. Muzigaba M, Van Wyk B, Puoane T. Management of severe acute malnutrition in children under 5 years through the lens of health care workers in two rural South African hospitals. African J Prim Hlth Care Family Med. 2018; 10:e1-e8.

14. Solnes Miltenburg A, Kiritta RF, Bishanga TB, van Roosmalen J, Stekelenburg J. Assessing emergency obstetric and newborn care: can performance indicators capture health system weaknesses? BMC Preg Childbirth. 2017; 17:92.

15. Ministry of Health and Family Welfare. Strengthening Facility Based Paediatric Care. Accessed on February 12, 2019. Available from Government of India; 2015.

16. Gathara D, Opiyo N, Wagai J, et al. Quality of hospital care for sick newborns and severely malnourished children in Kenya: a two-year descriptive study in 8 hospitals. BMC health services research. 2011;11:307.

17. Hategeka C, Shoveller J, Tuyisenge L, Lynd LD. Assessing process of paediatric care in a resource-limited setting: A cross-sectional audit of district hospitals in Rwanda. Paediatr and Int Child Hlth. 2018;38:137-45.

18. Enarson PM, Gie RP, Mwansambo CC, et al. Reducing deaths from severe pneumonia in children in Malawi by improving delivery of pneumonia case management. PLoS 
One. 2014;9:e102955-e.

19. Menge I, Esamai F, van Reken D, Anabwani G. Paediatric morbidity and mortality at the Eldoret District Hospital, Kenya. East African Med. J. 1995;72:165-9.

20. Irimu GW, Gathara D, Zurovac D, et al. Performance of health workers in the management of seriously sick children at a Kenyan tertiary hospital: before and after a training intervention. PloS One. 2012;7:e39964-e.

21. Mpimbaza A, Sears D, Sserwanga A, et al. Admission risk score to predict inpatient pediatric mortality at four public hospitals in Uganda. PLoS One. 2015;10:e0133950-e.

22. Ayieko P, Ntoburi S, Wagai J, et al. A multifaceted intervention to implement guidelines and improve admission paediatric care in Kenyan district hospitals: A cluster randomised trial. PLoS Med. 2011;8:e1001018-e.

23. Duke T, Tamburlini G. Improving the quality of paediatric care in peripheral hospitals in developing countries. Arch Dis Childhood. 2003;88:563-5.

\section{$\overline{\text { CLIPPINGS }}$}

Multi system inflammatory syndrome-children (MIS-C) Management (WHO/2019-nCoV/Sci_Brief) Multisystem_Syndrome_Children)

Management of MIS-C includes Immuno-modulatory, antiplatelet and anticoagulation therapy, Cardiac and Supportive management. A stepwise progression of immunomodulatory therapies should be used with IVIG $(2 \mathrm{gm} /$ $\mathrm{kg}$ ) considered first tier therapy. Low moderate dose glucocorticoids (1-2 mg/kg/day) should be given with IVIG as adjunctive therapy if shock and/or organ threatening disease present.

In patients not responding to IVIG and low moderate dose glucocorticoids, high dose, IV pulse glucocorticoids (10 $30 \mathrm{mg} /$ $\mathrm{kg} /$ day) may be considered. Anakinra ( $4 \mathrm{mg} / \mathrm{kg} /$ day IV or SQ) may be considered for treatment of MIS C refractory to IVIG and glucocorticoids. Anticoagulant anti platelet therapy in MIS$\mathrm{C}$ includes low dose aspirin (3-5 mg/kg/day; $\max 81 \mathrm{mg} /$ day), continued until normalization of platelet count and confirmed normal coronary arteries at $\geq 4$ weeks after diagnosis. Patients with abnormal BNP and/or troponin T at diagnosis should have these laboratory parameters trended over time until they normalize. ECGs should be performed at a minimum of every 48 hours in hospitalized patients and during follow up visits. Echocardiograms should be repeated at a minimum of 7-14 days and 4-6 weeks after presentation. Patients with left ventricular (LV) dysfunction and/or CAA will require more frequent echocardiograms.
Lung-protective ventilation in pediatric acute respiratory syndrome (PARDS) (Pediatr Allergy Immunol Pulmonol. 2019.35-44)

The goals of ARDS management are to treat the underlying cause, provide adequate oxygenation and ventilation, and protect the lungs from ventilator-induced lung injury (VILI). The aims of lung-protective ventilation are to avoid overdistension (volutrauma and barotrauma), minimize atelectrauma and minimize biotrauma.

Standard of care for mechanical ventilation in the PICU is generally consistent with the ARDS Network study, and the PALICC guidelines recommend tidal volumes of 3-6 and 5-8 $\mathrm{mL} / \mathrm{kg}$ for patients with poor and more preserved respiratory compliance, respectively, along with limiting inspiratory plateau pressure to $28 \mathrm{~cm} \mathrm{H} 2 \mathrm{O}$.

PALICC strongly recommends the use of PEEP up to $15 \mathrm{~cm}$ $\mathrm{H} 2 \mathrm{O}$ or greater for severe PARDS. To minimize the potential toxicity of ventilatory support required to oxygenate and ventilate PARDS patients, permissive hypoxemia and hypercapnia should be considered. PALICC also recommends oxygen saturation goals of $92 \%-97 \%$ for mild PARDS and $88 \%-92 \%$ and PEEP $>10 \mathrm{~cm} \mathrm{H} 2 \mathrm{O}$ for severe PARDS.

PALICC recommendations include considering permissive hypercapnia for moderate to severe PARDS to minimize VILI, maintaining $\mathrm{pH}$ 7.15-7.30 using lung-protective strategies.

CN RAGHUNATH raghu.picu@gmail.com 